\title{
Assessment of the accuracy of close distance photogrammetric JRC data
}

Authors: Dong Hyun Kim, George Poropat, Ivan Gratchev, Arumugam Balasubramaniam

\begin{abstract}
By using close range photogrammetry, this article investigates the accuracy of the photogrammetric estimation of rock joint roughness coefficients (JRC), a measure of the degree of roughness of rock joint surfaces. This methodology has proven to be convenient both in laboratory and site conditions. However, the accuracy and precision of roughness profiles obtained from photogrammetric 3D images have not been properly established due to the variances caused by factors such as measurement errors and systematic errors in photogrammetry.

In this study, the influences of camera-to-object distance, focal length and profile orientation on the accuracy of JRC values are investigated using several photogrammetry field surveys. Directional photogrammetric JRC data are compared with data derived from the measured profiles, so as to determine their accuracy. The extent of the accuracy of JRC values was examined based on the error models which were previously developed from laboratory tests and revised for better estimation in this study.

The results show that high resolution 3D images (point interval $\leq 1 \mathrm{~mm}$ ) can reduce the JRC errors obtained from field photogrammetric surveys. Using the high resolution images, the photogrammetric JRC values in the range of high oblique camera angles are highly consistent with the revised error models. Therefore, the analysis indicates that the revised error models facilitate the verification of the accuracy of photogrammetric JRC values.
\end{abstract}

Key words: photogrammetry, JRC, camera oblique angles, parabolic error models 


\section{List of Symbols}

$\alpha$ : pitch of a line on a plane measured from the strike of the plane

$\theta_{1}$ : angle between the line on a plane and the line of sight

$\theta_{2}$ : angle between the normal vector of a plane and the line of sight

$\mathrm{h}_{\mathrm{m}}$ : maximum asperity height measured by profile gauge

$h_{p}$ : maximum asperity height obtained from photogrammetric profiles

$\mathrm{JRC}_{0}$ : JRC values estimated based on manually measured profiles

$J_{R}$ : JRC values estimated based on photogrammetric profiles

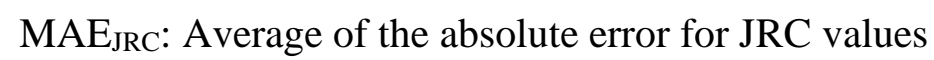

$$
M A E_{J R C}=\frac{\sum_{i=1}^{n}\left|J R C_{o, i}-J R C_{p, i}\right|}{N}
$$

$\mathrm{N}_{\text {JRC}}$ : Normalized JRC values comparing between photogrammetric profiles and manually measured data

$$
N_{J R C}=\frac{J R C_{3 D \text { images }}}{J R C_{\text {measured }}}
$$

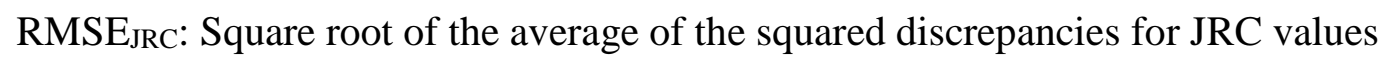

$$
R M S E_{J R C}=\sqrt{\frac{\sum_{i=1}^{n}\left(J R C_{o, i}-J R C_{p, i}\right)^{2}}{N}}
$$




\section{Introduction}

In rock engineering, joint roughness profiles and roughness parameters have been frequently investigated for the characterization of the shear strength of rock joints. As the most commonly used roughness parameter, rock joint roughness coefficients (JRC) are obtained through comparison of the manually measured surface geometry with standard profiles (Barton \& Choubey, 1977; ISRM, 1978). Recently, 3D joint roughness parameters have been devised to identify rock joint shear behaviour to provide a more realistic description of joint roughness distribution (Grasselli et al., 2002; Tatone \& Grasselli, 2010). Nevertheless, the majority of rock joint roughness analyses have employed linear profiles rather than 3D roughness parameters.

Close range photogrammetry (CRP) has been widely adopted to investigate many detailed aspects of rock slopes, as well as, large scale surveys in rock engineering and mining. The 3D models from CRP have been efficiently used for the mapping and characterization of large scale rock slopes due to the cost and labour advantages of digital photogrammetry. The recent development of high-end equipment for remote sensing surveys has also encouraged the spread of photogrammetry to obtain detailed surface roughness information such as rock joint roughness coefficients (JRC) and the joint set characteristics of rock slopes.

The applicability of close range photogrammetry (CRP) for the estimation of joint roughness from rock surfaces has previously been studied in various laboratory conditions and through photogrammetric surveys (Jessell et al., 1995; Cravero et al., 2001; Lee and Ahn, 2004; Unal et al., 2004; Bistacchi et al., 2011; Sturzenegger \& Stead, 2009). Several important studies, which focused on the possibility of JRC estimation using photogrammetric 3D models, presented the common result that high resolution images are required to obtain reliable JRC values from photogrammetry models (Haneberg, 2007; Baker et al., 2008; Poropat, 2008; Poropat, 2009). Consequently, these studies concur that the measurement accuracy of JRC data may be linearly reduced by increasing focal lengths and camera-to-object distances.

The uncertainty of the accuracy and precision of photogrammetric JRC values is derived from various factors. The influencing factors associated with the photogrammetric 3D models have been widely discussed by many researchers (Fraser, 1984; Poropat, 2009; Dai \& Lu, 2010; Fooladgar et al., 2013) and summarized by Dai et al. (2014). In terms of systematic errors, the influencing factors are categorized as camera and planning factors. Firstly, examples of the camera factors which lead to image based errors are lens distortion, principal distance and image resolution. The influence of the lens distortion and principal distance can be reduced by 
adjusting the relative parameters through a camera calibration procedure. The resolution of the image is directly controlled by the sensor size of the camera, the focal length of the lens employed and the camera-to-object distance. Secondly, planning factors include baseline distance, photograph overlap, angle of incidence and camera intersection angle, all of which represent the influence of camera network geometry on the accuracy of 3D models. In order to create accurate 3D images, these factors are controlled by following the recommendations of photogrammetry algorithms.

Based on the methodology of stereo photogrammetry, a JRC error model was developed (Kim et al., 2015a). This model demonstrates the relationships between the RMSE of photogrammetric JRC values and the proportion of over or underestimation of the JRC values considering camera-to-object distances. This model was created under an ideal laboratory condition by varying the camera-to-object distances and lens focal lengths. In this study, this error model is also investigated using both the RMSE and the mean absolute error (MAE) of JRCs to seek better correlations. The proposed error model can be used as a guideline for field photogrammetric JRC data which may involve additional errors induced by varying field conditions. Also, this model can be verified by analysing field photogrammetric JRC values.

In this study, the roughness data have been collected from surveys at six different sites performed over a period of three years. At each survey site, $2 \sim 8$ profiles were measured in accordance with the suitability and accessibility of the target surfaces for the measurements. This allows the measured profile shapes and their JRC values to be compared with the photogrammetric roughness data. The estimated JRC errors can be investigated based on the reconstructed error functions and the results of each field survey discussed with respect to the different site conditions. This study also investigates the influence of the oblique angles of the line of sight to the exposed rock surfaces in the photogrammetry setup, which is one of the most important differences compared to the controlled laboratory conditions.

\section{The use of close range photogrammetry in rock engineering}

Close range photogrammetry (CRP) has been widely used in geotechnical and geological engineering areas. A stereo pair or series of images are taken from consecutive positions for acquisition of 3D data. Through the use of CRP technique, slope models at large and small scale have been analysed for various engineering purposes. As a practical process, stereo photogrammetry has been performed using a variety of algorithms. For example, with specialized functions for the analysis of rock masses, the applications of the commercial- 
grade packages, Sirovision (CAE), 3DM Analyst (Birch, 2009) and ShapeMetrix3D (3G Software and Measurement), have all been described in civil and mining engineering (Haneberg, 2008). These packages share procedural similarities and their processes generally require specific positions and orientations of cameras as well as a pre-defined set of ground control points to achieve accurate 3D models. In this study, a Sirovision Version 5 (CSIRO, 2012) package is employed to create and analyse 3D models.

An alternative photogrammetric process is structure-from-motion (SfM). SfM has the advantage of being able to determine internal camera geometry, camera position and orientation automatically using consumer-grade digital cameras, as well as, potentially minimising distortions and generating dense point clouds by the use of multiple photographs with large overlap collected from different positions and directions. The SfM approach has found a variety of applications in the geosciences including surveys of geological structures, mine sites, landslides, volcanic edifices and glaciers (James \& Robson, 2012; Westoby et al., 2012; Fonstad et al., 2013; Bemis et al., 2014; Micheletti et al., 2015; Lato et al., 2015), with a flexibility that allows photogrammetric surveys to be conducted from unmanned aerial vehicles (UAVs) and kites (Niethammer et al., 2012; Vasuki et al., 2014; Bemis et al., 2014; James \& Robson, 2014; Ryan et al., 2015). In this study the advantage of CRP over SfM is the ability to generate a photogrammetric model from one stereo pair of images rather than requiring multiple angles and images, which is useful in regions of limited access.

\section{Directional roughness profiles and JRC values}

JRC values are estimated by using digitized profile data obtained from photogrammetric 3D models. In this study, using the extracted profiles with regular point intervals from 3D models, the coordinates can be used to calculate roughness parameters which are correlated with JRC values. Many researchers have presented mathematical correlations between roughness parameters and JRC values (Tse \& Cruden, 1979; Reeves, 1985; Franklin et al., 1988; Maerz et al., 1990; Yu \& Vayssade, 1991; Hsiung et al., 1993). Among these correlations, the roughness parameter, $\mathrm{Z}_{2}$ has shown strong relations with JRC values (Yu \& Vayssade, 1991). Tse \& Cruden (1979) originally established a regression equation using the $Z_{2}$ parameter, which is a discrete form as shown in Eq. (1), to characterize shapes of rock joint roughness profiles.

$$
Z_{2}=\left[\frac{1}{M\left(D_{x}\right)^{2}} \sum_{i=1}^{M}\left(y_{i+1}-y_{i}\right)^{2}\right]^{1 / 2}
$$




$$
J R C=32.2+32.47 \log Z_{2}
$$

where $\mathrm{M}$ is the number of intervals, $\mathrm{D}_{\mathrm{x}}$ is a constant distance lag and the sum of the squares in adjacent $y$-coordinates is divided by the product of the number of intervals. In this study, Eqs. (1) and (2) are employed to estimate JRC values using the coordinates of the obtained roughness profiles.

\section{Extraction of roughness profiles and points}

The use of data transformation to create roughness profiles for a specific direction has been well documented in the literature (Baker et. al., 2008; Haneberg, 2007, 2008). With the function of profile extraction from a surface model, the creation of profiles at positions of interest may be a complicated procedure and the selection of an area for positioning of profiles on the surface model can therefore also be inaccurate. Haneberg (2007) presented a useful method to produce roughness profiles in any direction by using a rotation and interpolation matrix. This interpolation can be performed by rotations based on the correlation between the azimuth of the dip-line of planes and the directions of profiles.

Focussing on the convenience of the comparisons between manually measured profiles and the corresponding profiles of 3D images, in this study we use a simplified method to extract roughness profiles from regularly gridded surface models by using point distances of roughness profiles from a guide line. It is assumed that the roughness profile is referenced by an imaginary line which links between the lowest two points of the line. The line lies on a more precise location of interest for the comparison of profiles. In the correlated locations between the roughness profile and the imaginary line, the roughness profile is defined by the shortest distances from the imaginary line as demonstrated in Figure 1. The shortest distances from the points to the guide line is estimated by Eq. (3) to (5). These equations and the general matrix form of point-line distance in three dimensional space are well demonstrated by Weisstein (2002).

In Fig. 1, $\mathrm{p}_{0}$ and $\mathrm{p}_{1}$, the vector along the line specified by two points is identified by Eq. (3) using their coordinates.

$$
\mathrm{v}=\left[\begin{array}{l}
x_{0}+\left(x_{1}-x_{0}\right) t \\
y_{0}+\left(x_{1}-x_{0}\right) t \\
z_{0}+\left(x_{1}-x_{0}\right) t
\end{array}\right]
$$

The shortest distances between the points and an imaginary line between $\mathrm{p}_{0}$ and $\mathrm{p}_{1}$ with parameter t are estimated by Eq. (4).

$d^{2}=\left[\left(x_{0}-x_{n}\right)+\left(x_{1}-x_{0}\right) t\right]^{2}+\left[\left(y_{0}-y_{n}\right)+\left(y_{1}-y_{0}\right) t\right]^{2}+\left[\left(z_{0}-z_{n}\right)+\left(z_{1}-z_{0}\right) t\right]^{2}$ 
The parameter $t$ is obtained by Eq. (5):

$$
\mathrm{t}=-\frac{\left(p_{0}-p_{n}\right) \cdot\left(p_{1}-p_{0}\right)}{\left|p_{1}-p_{0}\right|^{2}}
$$

The results of the calculations are generated using AutoCAD Civil 3D (Autodesk, 2011) to calculate the JRC values.

\section{Evaluation of the accuracy of photogrammetric JRC values}

To measure the accuracy of continuous variables, the root-mean-squared-error (RMSE) and the mean absolute error (MAE) are commonly employed. RMSE has been widely used to identify the accuracy of data due to its high correlation between the predicted values and the observed values. In a photogrammetry standard, the accuracy of geospatial data obtained from photogrammetry has been classified using the RMSE of data coordinates (ASPRS, 2014). As a natural measure of average error magnitude, the advantages of MAE have also been reported (Willmott \& Matsuura, 2005; Chai \& Draxler, 2014).

A parabolic error model, which describes the RMSE of photogrammetric JRC values according to camera-to-object distances, was developed in a previous laboratory study (Kim et al., 2015a). Using the quadratic error functions, the ranges of JRC errors obtained from the employed focal lengths and camera-to-object distances can be illustrated by the sizes of the parabolas. The quadratic equations were derived from a set of laboratory photogrammetry tests using three different focal length lenses ( $F L=24,50,85 \mathrm{~mm}$ ) in an ideal laboratory condition. The photographs of a rock sample were taken with the perpendicular camera orientation to the object and keeping the same indoor lighting conditions. The background of the developed models is detailed in the literature (Kim et al., 2015a).

As shown in Fig. 2 (a), RMSEJRC, together with camera-to-object distances, form quadratic functions using the normalized JRC values. The basic form of the quadratic functions used is presented in Eq. (6) to (8), where:

$$
\begin{aligned}
& R M S E_{J R C}=\sqrt{\frac{\sum_{i=1}^{n}\left(J R C_{0, i}-J R C_{p, i}\right)^{2}}{N}} \\
& N_{J R C}=\frac{J R C_{3 D \text { images }}}{J R C_{\text {measured }}} \\
& R M S E_{J R C} \cdot D=a N_{J R C}^{2}+b N_{J R C}+c
\end{aligned}
$$


where $J R C_{o}$ is the manually measured JRC value and $J R C_{p}$ is the JRC values obtained from the photogrammetry models of each profile. $N_{J R C}$ is a normalized value which indicates the accuracy of the photogrammetric JRC values compared to manual measurements. In Eq. (8), ' $\mathrm{D}$ ' represents the camera-to-object distance and ' $a$ ' is a coefficient to determine the direction and size of the parabola which represents the precision of the data; and ' $b$ ' and ' $c$ ' are the coefficients for the locations of the vertex of the parabolic curves. The proposed quadratic functions show upward parabolic curves with different widths according to the focal length of lenses. However, since the laboratory data are predominantly plotted in the range of underestimation, the quadratic regression curves in the range of overestimation may not accurately describe the correlations.

Considering the balance of data, it is reasonable that the data can be interpreted by dividing the data range into underestimation and overestimation categories. In this study, the JRC data obtained from the laboratory tests reconstructed error functions using MAE of the JRC values is shown in Fig. 2 (b). With the use of absolute values, the basic form of the MAE of JRC is simpler than the RMSE form, as given in Eq. (9).

$$
M A E_{J R C}=\frac{\sum_{i=1}^{n}\left|J R C_{o, i}-J R C_{p, i}\right|}{N}
$$

In the total data range, the overall patterns of the relationship between RMSE or MAE and $\mathrm{N}_{\text {JRC }}$ are similar showing quadratic regression lines. However, in the overestimation range, linear regressions between $\mathrm{MAE}_{J R C}$ and $\mathrm{N}_{\mathrm{JRC}}$ are formed, displaying high values of the coefficient of determination $\left(\mathrm{R}^{2}=0.79\right.$ to 0.96$)$. The large focal lengths create steeper inclinations of the regression line than the short focal lengths. Similarly, in the underestimation category data range, the quadratic regressions of RMSE $\mathrm{JRC}_{\text {show better }}$ correlations with $\mathrm{N}_{\mathrm{JRC}}$ than MAEJRC and the previous parabolas. The improved relationships are shown by the $\mathrm{R}^{2}$ values, as given in Fig. 2 (a) and (b).

Chai and Draxler (2014) stated that MAE is appropriate to explain uniformly distributed errors, and RMSE is better for describing a normal distribution. Similarly, the distinct characteristics between MAE and RMSE have also been reflected in the present study. With respect to data distributions, it is interesting that MAE is suitable to describe the data in the overestimation range, and RMSE is good for describing the underestimated data range in Fig. 2 (b). This may be quantitatively explained by the wide differences in the total number of data in both ranges. Qualitatively, the data distribution might also be affected by the source of the errors. It is because the underestimation is mainly derived from the insufficient density of the 3D images, while the overestimation is predominantly caused by data distortion and data noise. 


\section{Field photogrammetry surveys}

\subsection{Geological condition of the study areas}

Photogrammetry surveys were performed at six different sites on the Gold Coast and in Brisbane, Australia. The reported surveys in this study were carried out over a period of 3 years from 2013 to 2015. The selected sites are road cuts, an excavated slope and a landscaping site. The rock masses are composed of shale, sandstone, metasandstone and greywacke from the Neranleigh-Fernvale beds (Willmott, 2010). The surface conditions of the target areas varied including exposed bedding planes, joint planes and individual block surfaces. With consideration for the practice of manual measurement of roughness profiles, the target areas were determined focusing on the photograph conditions such as height and camera-to-object distances.

The geological conditions at the selected sites are summarized in Table 1 . The target areas at site \#1 comprise clearly exposed bedding planes of shale and sandstone joints. The orientations of the surfaces were mostly slanted against the camera axis. The target areas at site \#2 included parts of an exposed joint surface of shale and the road cuts were partly covered by plants. The location of the target area was lower than the camera height. The target area at site \#3 was clearly exposed by excavation and was a weathered joint surface. The target areas at sites \#4 and \#5 were parts of road cuts and the exposed joint planes were almost orthogonally oriented to the optical axis. The target areas at site \#6 were parts of sandstone rock blocks for landscaping which were individually oriented with different angles to the optical axes. Detailed geological conditions and strength properties for the sandstone and shale at the sites are presented in the previous literature by the authors (Kim et al., 2013, 2015b).

\subsection{Planning for minimizing photogrammetry errors}

Photogrammetry surveys often require the use of high-end cameras and long focal length lenses to create high resolution images. However, other factors when designing photogrammetry layout also influence the accuracy of 3D models. In the case of stereoscopic photographs, it is recommended that the image pairs overlap each other by more than $60 \%$ to create the best 3D images (CSIRO, 2012). The range of base-to-distance ratio is also recommended relating to the depth accuracy of the 3D images. Generally, the image based errors are caused by lens distortion and the errors are usually minimized by employing 
calibration procedures. Accordingly, the employed lenses in this study were fully compatible with the photogrammetry software, Sirovision. The base-to-distance (B:D) ratio was also fixed at the ratio of 1:7 based on the desirable range of the program (CSIRO, 2012).

In addition, orthogonal images for the object create more accurate 3D images. However, for a region of natural rock surfaces, the line of sight of the camera is usually oblique to the area being imaged. As high-angle oblique photographs are desirable for accuracy, the orientations of the profiles and the surfaces should be considered for data interpretation. In this study, the stereo image pairs were taken towards the slope with levelled camera view angles (tilting angle was $0^{\circ}$ ). Thus, the orientations of the profiles of interest on rock surfaces were conveniently interpreted based on a horizontal line of sight.

\subsection{Data collection}

Two stereo images were obtained at each camera position using a Nikon D7000 digital camera (sensor resolution: $4928 \times 3624$ pixels) with fixed focal length lenses (Nikon Nikkor, $\mathrm{FL}=24,50,85 \mathrm{~mm})$. The summaries of the photogrammetry setup at the sites are presented in Table 2. Through the site surveys, the range of employed camera-to-object (c-to-o) distances varied from 2 to $33 \mathrm{~m}$. The specific sections of the rock slopes were marked using circular or rectangular targets which were 30 to $50 \mathrm{~cm}$ apart as shown in Fig. 3. The targets were arranged into square, triangle or line shapes according to the measurement availability. Roughness profiles were manually measured using a profile gauge (1 mm interval) for the lines between targets. For all six site surveys, photographs were taken on bright sunny days and the target areas of the slopes were unaffected by shadows.

3D images were created using Sirovision. In order to reduce a possible error due to camera factors, the calibrations of the employed lenses and camera body combinations were performed using the calibration data files provided by the manufacturer. The point cloud intervals of the 3D models generally increased with the camera-to-object distances and the intervals were inversely proportional to the lens focal length employed. However, the points in space created by a 3D image were inconsistent due to the irregularity of the natural slope surfaces. This appearance was heightened by the fact that the surface being imaged is not flat, so the points appear to be scattered in an irregular pattern.

\section{Accuracy of photogrammetric roughness data}

\subsection{Profile agreement}


A total of 30 measured profiles were compared with the photogrammetric profiles. The photogrammetric roughness profiles were generated from the 3D data based on the point-toline distance method as described in section 4. Overall, a photogrammetry setup at close distance with longer focal length lenses created well matched profiles. Fig. 4 presents an example of the profile deviations of site \#4 in accordance with the employed focal length of the lenses. The vertical scale is exaggerated two times for clarity. Due to insufficient data points in the 3D models ( $8.7 \mathrm{~mm}$ point cloud distance), the profile which has been created from the $24 \mathrm{~mm}$ lens, showed considerable mismatch as shown in Fig 4 where the agreement of the profile shapes is governed by the point interval of 3D images. With smaller point intervals ( 2.2 to $4.0 \mathrm{~mm}$ ), the large scale undulation in the profiles obtained from both the 50 $\mathrm{mm}$ and $85 \mathrm{~mm}$ lenses is comparatively consistent with the measured profiles (see Fig. 4 (b) and (c)). The accuracy of the profiles in a 3D model can also vary with the locations of the sections of interest due to the irregular pattern of the originally formed mesh, as mentioned in section 6. As demonstrated in Fig. 5, the angles between the line of sight and the normal vector of a rock surface vary with the sections of interest in an image. Consequently, the point cloud intervals of the 3 sections varied from $12.8 \mathrm{~mm}$ to $22.4 \mathrm{~mm}$ in the same 3D model.

In Fig. 6 (a), the obtained data on point cloud intervals from the field surveys generally shows a gradual increase with camera-to-object distance. The point intervals show rather scattered patterns $\left(\mathrm{R}^{2}=0.66\right.$ to 0.84$)$ compared to those obtained under laboratory conditions obtained in a previous study by the authors (Kim et al., 2015a). The dispersion of data is clearly shown from the results obtained using the short focal length $(24 \mathrm{~mm})$. Under laboratory conditions, based on orthogonal images, a linear increase of point intervals in the 3D images was obtained with increasing camera-to-object distances with $\mathrm{R}^{2}=0.95$ to 0.99 as shown in Fig. 6 (b). This reflects the irregularity of the rock surface orientations for the line of sight of the camera setup and may also explain the reason why the irregular point intervals can be attributed to the influence of the variation of the rock surface orientations.

The oblique angle of the camera setup facing a rock slope can influence the accuracy of photogrammetric profiles. This issue was examined by Hong et al. (2008) who found that inclined sample surfaces can produce underestimated roughness data. In a general orthogonal photograph setup, the oblique angle of the line of sight to a plane can be identified by the angle between the line of sight and the line of interest on a plane. As shown in Fig. 7 (a), the angles are illustrated by both the angle between the line of interest on a plane and the optical axis $\left(\theta_{1}^{\circ}\right)$ and the angle between the optical axis and the normal vector of the plane $\left(\theta_{2}{ }^{\circ}\right)$. These angles can be represented on a stereographic projection which is a convenient tool to 
identify geological structures on rock faces in any orientations. The method of the hemispherical projection is well described by Priest (1985) and Goodman (1989).

With the strike of a plane, the angle of pitch is measured from the perimeter of the net along the great circle. In Fig. 7 (b), line 1 in the plane 'A' has a pitch of $\alpha^{\circ}$ measured from the strike line. The acute angle between the optical axis and the given line 1 is counted from the pitch $\theta_{1}{ }^{\circ}$. In a similar way, $\theta_{2}^{\circ}$ is counted from the perimeter between the optical axis and the pole of plane ' $\mathrm{A}$ ' along the great circle of plane 'B' containing the line 1. Accordingly, discrepancies in photogrammetric profiles could occur as $\theta_{1}^{\circ}$ decreases and as $\theta_{2}^{\circ}$ increases.

In this study, the deviation of the profile waviness between manual measurement and photogrammetry was simply quantified by using the differences in the maximum asperity heights between 3D models $\left(h_{p}\right)$ and manual measurement $\left(h_{m}\right)$. For all measured roughness profiles, $h_{p}$ and $h_{m}$ values were obtained using the same profile length $(L=30 \mathrm{~cm}$ ) based on the straight edge method. This method has been used for roughness estimation in large scale profiles (Piteau, 1970; Milne et al., 1992; Palmström, 2001). By comparing the manually measured profiles with the photogrammetric profiles, the accuracy of profile waviness was investigated using the deviation ratio of the maximum asperity height $\left(h_{p}-h_{m} / h_{m}\right)$ and the results plotted according to the point intervals in Fig. 8. When the values are close to ' 0 ', better agreement between the manual profiles and the photogrammetric profiles are achieved. The field data show scattered distributions, however, the data in the range of less than $1 \mathrm{~mm}$ intervals tend to be close to the mean values with a lower standard deviation ( $\mathrm{SD}=0.33$, Fig. 8 (a)) than the data in the range of more than $1 \mathrm{~mm}$ point interval (SD = 0.76, Fig. 8 (b)).

It was also found that high oblique angles for the camera axis could reduce the degree of dispersion of photogrammetric profiles. The data plotted in Fig. 8 (a) and (b) are sorted by the high-angle oblique range of $\theta_{1}>60^{\circ}$ and $\theta_{2}<30^{\circ}$. These ranges indicate that the angles between the camera axes and the profiles are acute close to the orthogonal photographs within the range of $60^{\circ} \sim 90^{\circ}$. As shown in Fig. 8 (c) and (d), within these data ranges, the variations of the data are obviously reduced from 0.16 to 0.13 (point interval $\leq 1.0 \mathrm{~mm}$ ) and from 0.76 to 0.34 (point interval $>1.0 \mathrm{~mm}$ ). The result indicates that the accuracy of photogrammetric profiles is affected by the oblique angles $\theta_{1}$ and $\theta_{2}$ and orthogonal photographs are appropriate for the measurement of roughness.

\subsection{Accuracy and precision of photogrammetric JRC values}

JRC values were estimated by using the small scale asperity heights of the photogrammetric profiles, as demonstrated in Eq. (1) and (2). A total of 111 JRC values were used to analyse 
the results. The obtained photogrammetric JRC values are summarized in Table 4 and the results of each site are demonstrated in Fig. 9 and Fig. 10. According to the site conditions, the obtained JRC values showed different trends. The JRC values for sites \#1 and \#3, which were obtained using a short focal length lens $(\mathrm{FL}=24 \mathrm{~mm})$, from a large c-to-o distances (17 m, $33 \mathrm{~m}$ ), were mostly underestimated due to insufficient data points (Fig. 9 (a) and (c)). It was observed that some of the 3D images at site \#2 were influenced by plants near the target areas. Consequently, the low resolution images which were taken by the $24 \mathrm{~mm}$ lens created distorted 3D models and resulted in overestimations of JRC values (Fig. 9 (b)). This tendency was similarly found for sites \#4 and \#5. The target areas in these sites were clearly exposed and orthogonally oriented to the camera axes and the differences of JRCs between manual and photogrammetry were relatively less than at other sites. However, large differences between the JRCs are found for low resolution images (FL=24 mm), as shown in Fig. 9 (d) and (e).

Fig. 10 demonstrated the variation of JRC values for site \#6 for different focal lengths. In contrast to the other sites, the photogrammetric 3D images mainly overestimated the JRC values. Also, in some profiles, large variations in JRC values were found throughout the camera-to-object distance range when $24 \mathrm{~mm}$ and $50 \mathrm{~mm}$ lenses were used. These overestimated values were predominantly due to distorted waviness in the extracted profiles. The use of a longer focal length lens (FL $=85 \mathrm{~mm}$ ) reduced the data deviation (Fig.10 (c)).

The JRC data are plotted and compared with the error models for both the ranges of underestimation and overestimation in Fig. 11. In contrast to the authors' previous laboratory study (Kim et al., 2015a), it appears that the plotted data are quite dispersed according to each field survey condition and mostly distributed in the overestimated region, as demonstrated in Fig. 11. Overestimation can be found in both the high resolution and low resolution images. The reasons are due to the influence of data noise as discussed by Poropat (2008), the distortion of waviness in the profiles leading to an overestimation of JRCs at low resolutions. As shown in Fig. 11 a) c), the data also deviate from the error models with the deviations more obvious when shorter focal length lenses are employed. This deviation may be attributed to various sources of errors derived from different site conditions. However, we suggest that the differences are mainly due to the lack of image resolution. In the range of high resolution point intervals (less than $1 \mathrm{~mm}$ ), the deviations are considerably reduced, as demonstrated in Fig. $11 \mathrm{~d}) \sim \mathrm{f}$ ).

It is observed that the deviation is reduced within the range of high oblique angles (e.g. $\theta_{1}>$ $60^{\circ}$ and $\theta_{2}<30^{\circ}$ ). The black dots in Fig. $\left.11 \mathrm{~d}\right) \sim$ e) show that the sorted data are closely distributed around the error curves. This distribution shows that the orthogonal photographs 
improve the accuracy of photogrammetric JRCs as evident from the trends of the data for each focal length. As a consequence, the data distributions obtained from the site photogrammetry survey indicate that the oblique camera angles can influence the accuracy of both the waviness of profiles in large scale roughness and the deviations of JRC values in small scale roughness. It should be also noted that the results of data analysis could be influenced by the inconsistency in the amount of data recorded at each field survey. Further studies using more data from varied field conditions will allow improved understanding of the proposed error models.

\section{Discussion}

A combination of large sensor sizes and longer focal lengths can achieve high resolution images of joint surfaces. This combination can show linear relationships between the camera combinations and camera-to-object distances under laboratory conditions. However, under field conditions the point intervals of the obtained 3D images vary with the complexity of the object. This study demonstrates that the point interval is an important factor and determines the level of accuracy of photogrammetric JRC data. For the same measurement interval with manual measurements $(\approx 1.0 \mathrm{~mm})$, this study showed the feasibility of stereo photogrammetry for JRC estimation. Within the range of point interval $(\leq 1.0 \mathrm{~mm})$, the errors could be interpreted using the developed error models with close correlations.

Unlike laboratory conditions, the field photogrammetry surveys which were performed under different conditions, resulted in scattered data and mainly overestimated JRC values. The obtained JRC values sometimes showed large discrepancies due to distortion of 3D models and data noise. A comparison of the results from previous lab tests and field surveys is summarized in Table 4. The most important factor influencing the accuracy of 3D models is an image error due to obstacles in the target areas, such as surrounding plants, large voids and rock fragments. The existence of these factors causes a large distortion of the 3D images and results in the overestimation of JRC values. In the laboratory conditions, the range of JRC values was predominantly underestimated. In such cases, distortion of 3D models was rarely observed while the undulations of profiles were insufficiently represented due to a lack of points.

The results of the field tests also showed the influence of the profile orientation. To obtain better accuracy, orthogonal photographs are recommended. This study showed the effect of the photograph angles on the accuracy of roughness data within the range of high oblique 
angles (e.g. $\theta_{1}>60^{\circ}$ and $\theta_{2}<30^{\circ}$ ). In practice, this methodology can be an issue for tripod based stereo photogrammetry. The limitation of the camera angles can be offset by using SfM which is a more flexible photogrammetry setup, the feasibility of which can be investigated in future research.

\section{Conclusions}

Photogrammetric roughness data were investigated under field conditions. Linear profiles and photogrammetric JRC values were obtained from the 3D models, and the accuracy of the results investigated using the proposed error models focussing on the influence of data intervals and orientation of the profiles. A total of 30 profiles were manually measured and compared with the 3D models created in accordance with varying camera-to-object distances and lens focal length. Based on the field data, the following conclusions can be drawn:

- This study improved the previous JRC error models by using better correlations, in the overestimation range, between $\mathrm{MAEJRC}_{\mathrm{J}} \cdot \mathrm{d}$ and $\mathrm{N}_{\mathrm{JRC}}$.

- In comparison with the error models, the JRC values of the field data were generally overestimated and showed considerable scatters. The most influential of all the factors on the accuracy of roughness data was the point interval of 3D models; a data range of 1 $\mathrm{mm}$ can reduce the differences from the error models.

- The field data also show that the oblique angles of the optical axis to the pole $\left(\theta_{1}\right)$ and to the measured profile $\left(\theta_{2}\right)$ can significantly affect the accuracy of both the maximum asperity height and JRC values of the profiles. The oblique angles could be usefully interpreted by stereographic projection methods. In the range of the oblique angles $\left(\theta_{1}>\right.$ $60^{\circ}$ and $\theta_{2}<30^{\circ}$ ), which are close to orthogonal, photogrammetry setups could improve the accuracy of the maximum asperity heights $\left(\mathrm{h}_{\mathrm{p}}\right)$ and JRC values. This shows that stereo photogrammetry can be used to produce linear roughness profiles and JRC estimation in a specific photographic setup.

\section{Acknowledgements}

This research was performed with the financial support of the Griffith University International Postgraduate Research Scholarship (GUIPRS). The authors would like to express their appreciation to CSIRO for providing the program SIROVISION for this study.

\section{References}


American Society for Photogrammetry \& Remote Sensing (2014) ASPRS positional accuracy standards for digital geospatial data. PE \& RS 81(3): A1-A26

Autodesk (2011) AutoCAD Civil3D 2011 User’s Guide, Autodesk, Inc. San Rafael, USA

Baker BR, Gessner K, Holden EJ, Squelch A (2008) Automatic detection of anisotropic features on rock surfaces. Geosphere 2008 4(2): 418-428

Barton N, Choubey V (1977) The shear strength of rock joints in theory and practice. Rock Mechanics 10: 1-54

Bemis SP, Micklethwaite S, Turner D, James MR, Akciz S, Thiele ST, Bangash HA (2014) Ground-based and UAV-based photogrammetry: A multi-scale, high-resolution mapping tool for structural geology and paleoseismology. J Struct Geol 69: 163-178

Birch JS (2009) Using 3DM Analyst mine mapping suite for slope stability-case studies. In: Proceedings of ACG Geotechnical Engineering for open pit mines, Section 17, pp.1-15

Bistacchi A, Griffith WA, Smith SAF, Di Toro G, Jones R, Nielsen S (2011) Fault roughness at seismogenic depths from LIDAR and photogrammetric analysis. Pure Appl Geophys, 168: $2345-2363$

Chai T, Draxler RR (2014) Root mean square error (RMSE) or mean absolute error (MAE)?Arguments against avoiding RMSE in the literature. Geosci Model Dev, 7: 1247-1250

Cravero M, Iabichino G, Ferrero AM (2001) Evaluation of joint roughness and dilatancy of schistosity joints. In: Proceedings of Eurock 2001, Espoo, pp 217-222

CSIRO (2012) Siro3D - 3D imaging system manual, Version 5.0, CCSIRO Exploration \& Mining, www.sirovision.com

Dai F, Lu M (2010) Assessing the accuracy of applying photogrammetry to take geometric measurements on building products. J Constr Eng Manag 136: 242-225

Dai F, Feng Y, Hough R (2014) Photogrammetric error sources and impacts on modelling and surveying in construction engineering applications. Vis Eng 2: 1-14

Fonstad MA, Dietrich JT, Courville BC, Jensen JL, Carbonneau PE (2013) Topographic structure from motion: a new development in photogrammetric measurement. Earth Surf Process Landforms 38: 421-430

Fooladgar F, Samavi S, Soroushmehr SMR, Shirani S (2013) Geometrical analysis of localization error in stereo vision systems. IEEE Sens J 13(11): 4236-4246

Franklin JA, Maerz NH, Bennett CP (1988) Rock mass characterization using photoanalysis. Int J Min Geol Eng 6: 97-112 
Fraser CS (1984) Network design considerations for non-topographic photogrammetry. Photogramm Eng Remote Sensing 50(8): 1115-1126

Goodman RE (1989) Introduction to rock mechanics. John Wiley \& Sons, New York

Grasselli G, Wirth J, Egger P (2002) Quantitative three-dimensional description of a rough surface and parameter evolution with shearing. Int J Rock Mech Min Sci 39: 789-800

Haneberg WC (2007) Directional roughness profiles from three-dimensional photogrammetric or laser scanner point clouds. In: Proceedings of the 1st Canada-U.S. rock mechanics symposium, Vancouver, pp 101-106

Haneberg WC (2008) Using close range terrestrial digital photogrammetry for 3-D rock slope modeling and discontinuity mapping in the United States. Bull Eng Geol Environ 67: 457469

Hong ES, Lee JS, Lee IM (2008) Underestimation of roughness in rough rock joints. Int J Numer Anal Met 32: 1385-1403

Hsiung SM, Ghosh A, Ahola MP, Chowdhury AH (1993) Assessment of conventional methodologies for joint roughness coefficient determination. Int J Rock Mech Min Sci Geomech Abstr 30(7): 329-343

ISRM (1978) Suggested methods for the quantitative description of discontinuities in rock masses. Int J Rock Mech Min Sci Geomech Abstr 15: 319-368

James MR, Robson S (2012) Straightforward reconstruction of 3D surface and topography with a camera: Accuracy and geoscience application. J Geophys Res 117: F03017

James MR, Robson S (2014) Mitigating systematic error in topographic models derived from UAV and ground-based image networks. Earth Surf Process Landforms 39: 1413-1420

Jessell MW, Cox SJD, Schwarze P, Power W (1995) The anisotropy of surface roughness measured using a digital photogrammetric technique. Fractography: fracture topography as a tool in fracture mechanics and stress analysis, Geological Society Special Publication 92: $27-37$

Kim DH, Gratchev I, Balasubramaniam AS (2013) Determination of joint roughness coefficient (JRC) for slope stability analysis: a case study from the Gold Coast area. Landslides 10: 657-664

Kim DH, Poropat GV, Gratchev I, Balasubramaniam AS (2015a) Improvement of photogrammetric JRC data distributions based on parabolic error models. Int J Rock Mech Min Sci 80: 19-30 
Kim DH, Gratchev I, Berends J, Balasubramaniam AS (2015b) Calibration of restitution coefficients using rockfall simulations based on 3D photogrammetry model: a case study. Nat Hazards 78: 1931-1946

Lato M, Gauthier D, Quinn P, Hutchinson DJ, Kromer RA, Edwards T, Riopel J (2015) 3D data collection for rapid rock fall response situations. In: Proceedings of GEOQuébec 2015, Canada, p513

Lee HS, Ahn KW (2004) A prototype of digital photogrammetric algorithm for estimating roughness of rock surface. Geosci J 8(3): 333-341

Maerz NH, Franklin JA, Bennett CP (1990) Joint roughness measurement using shadow profilometry. Int J Rock Mech Min Sci Geomech Abstr 27: 329-343

Micheletti N, Chandler JH, Lane SN (2015) Structure from Motion (SfM) photogrammetry. Geomorphological Techniques, Chap. 2, Sec. 2.2, ISSN 2047-0371

Milne D, Germain P, Potvin Y (1992) Measurement of rock mass properties for mine design. In: Proceedings of the ISRM-Eurock symposium on rock characterization, A.A. Balkema, Chester, England, pp 245-250

Niethammer U, James MR, Rothmund S, Travelletti J, Joswig M (2012) UAV-based remote sensing of the Super-Sauze landslide: Evaluation and results. Eng Geol 128: 2-11

Palmström A (2001) In-situ characterization of rocks. Lise, Abingdon, Exton (PA), Tokyo: A.A. Balkema Publishers, 2001

Piteau DR (1970) Geological factors significant to the stability of slopes cut in rock. In: Proceedings of the on planning open pit mines, Johannesburg, South Africa, pp 33-53

Poropat GV (2008) Remote characterization of surface roughness of rock discontinuities. In:

Proceedings of the 1st Southern hemisphere international rock mechanics symposium, Perth, Australia, Potvin Yves, CSIRO, Curtin University of Technology, University of Western Australia et al., pp 447-458

Poropat GV (2009) Measurement of surface roughness of rock discontinuities. In: Proceedings of the $3^{\text {rd }}$ CANUS rock mechanics symposium, Toronto, May 2009, Paper 3976

Priest SD (1985) Hemispherical projection methods in rock mechanics. George Allen \& Unwin, London.

Reeves MJ (1985) Rock surface roughness and friction strength. Int J Rock Mech Min Sci Geomech Abstr 22(6): 429-442 
Ryan JC, Hubbard AL, Box JE, Todd J, Christoffersen P, Carr JR, Holt TO, Snooke N (2015) UAV photogrammetry and structure from motion to assess calving dynamics at Store Glacier, a large outlet draining the Greenland ice sheet. The Cryosphere 9: 1-11

Sturzenegger M, Stead D (2009) Close-range terrestrial digital photogrammetry and terrestrial laser scanning for discontinuity characterization on rock cuts. Eng Geol 106: 163-182

Tatone BSA, Grasselli G (2010) A new 2D discontinuity roughness parameter and its correlation with JRC. Int J Rock Mech Min Sci 47: 1391-1400

Tse R, Cruden DM (1979) Estimating joint roughness coefficients. Int J Rock Mech Min Sci Geomech Abstr 16: 303-307

Unal M, Yakar M, Yildiz F (2004) Discontinuity surface roughness measurement techniques and the evaluation of digital photogrammetric method. In: Proceedings of the $20^{\text {th }}$ International congress for photogrammetry and remote sensing, ISPRS, pp 1103-1108

Vasuki Y, Holden EJ, Kovesi P, Micklethwaite S (2014) Semi-automatic mapping of geological structures using UAV-based photogrammetric data: An image analysis approach. Comput Geosci 69: 22-32

Weisstein EW (2002) Point-Line Distance--3-Dimensional. MathWorld, A Wolfram Web Resource. http://mathworld.wolfram.com/Point-LineDistance3-Dimensional.html

Westoby MJ, Brasington J, Glasser NF, Hambrey MJ, Reynolds JM (2012) 'Structure-fromMotion' photogrammetry: A low-coast, effective tool for geoscience applications. Geomorphology 179: 300-314

Wickens EH, Barton NR (1971) The application of photogrammetry to the stability of excavated rock slopes. Photogrammetric Record 7(37): 46-54

Willmott WF (2010) Rocks and landscape of the Gold Coast Hinterland. Geological Society of Australia, Queensland division.

Willmott CJ, Matsuura K (2005) Advantages of the mean absolute error (MAE) over the root mean square error (RMSE) in assessing average model performance. Clim Res 30: 79-82

Yu X, Vayssade B (1991) Joint profiles and their roughness parameters. Int J Rock Mech Min Sci Geomech Abst 28(4): 333-336 


\section{List of Figures}

Fig. 1 Coordinates of a roughness profile in three dimensional space

Fig. 2 JRC error models based on lens focal length in laboratory conditions: RMSE parabola models (Kim et al., 2015a) (a), revised RMSE and MAE error models (b)

Fig. 3 Slope photographs and attached targets for roughness measurement: at site \#1 (Beaudesert-Nerang road) (a), site \#3 (Bethania) (b)

Fig. 4 Comparison of a roughness profile at site \#4 between manual measurement and 3D models according to the employed focal length (FL): $24 \mathrm{~mm}$ (a), $50 \mathrm{~mm}$ (b), $85 \mathrm{~mm}$ (c)

Fig. 5 A window image from Sirovision and the sections of interest (Site \#1, C-to-O distance: $17 \mathrm{~m}, \mathrm{FL}=24 \mathrm{~mm}$ )

Fig. 6 Variation of point cloud intervals of 3D images: site investigation (a), laboratory condition (Kim et al., 2015a) (b)

Fig. 7 Camera oblique angle and normal vector of a section of interest: in 3D space (a), stereographic projection (b)

Fig. 8 Distribution of maximum asperity height ratio according to point cloud intervals: total data, point interval $\leq 1.0 \mathrm{~mm}$ (a), total data, point interval $>1.0 \mathrm{~mm}$ (b), data within the high-angle oblique $\left(\theta_{1}>60^{\circ}\right.$ and $\left.\theta_{2}<30^{\circ}\right)$, point interval $\leq 1.0 \mathrm{~mm}$ (c) and data within the high-angle oblique $\left(\theta_{1}>60^{\circ}\right.$ and $\left.\theta_{2}<30^{\circ}\right)$, point interval $>1.0 \mathrm{~mm}$ (d)

Fig. 9 Comparison of JRC values between manual and photogrammetry in site \#1 (a), site \#2 (b), site \#3 (c), site \#4 (d), site \#5 (e)

Fig. 10 Comparison of JRC values between manual and photogrammetry in site \#6: FL= 24 $\mathrm{mm}$ (a), FL = $50 \mathrm{~mm}$ (b), FL = $85 \mathrm{~mm}$

Fig. 11 Distribution of JRC error data and comparison to the error models: total data of FL = $24 \mathrm{~mm}$ (a), FL = $50 \mathrm{~mm}$ (b), FL = $85 \mathrm{~mm}$ (c) and the data of point intervals $\leq 1 \mathrm{~mm}$ of $\mathrm{FL}=24 \mathrm{~mm}(\mathrm{~d}), \mathrm{FL}=50 \mathrm{~mm}(\mathrm{e}), \mathrm{FL}=85 \mathrm{~mm}(\mathrm{f})$ 